\title{
Observing the Galactic Plane with the Cherenkov Telescope Array
}

\section{CTA consortium represented by Roberta Zanin ${ }^{*}$ and Jamie Holder ${ }^{\ddagger}$}

E-mail: robertazanin@gmail.com

The Cherenkov Telescope Array is a next generation ground-based gamma-ray observatory designed to detect photons in the $20 \mathrm{GeV}$ to $300 \mathrm{TeV}$ energy range. With a sensitivity improvement of up to one order of magnitude on the entire energy range with respect to currently operating facilities, coupled with significantly better angular resolution, the array will be used to address many open questions in high-energy astrophysics. In addition, CTA will explore the ultra-high energy $(\mathrm{E}>50 \mathrm{TeV})$ window with great sensitivity for the first time.

CTA is expected to reveal a detailed picture of the Galactic plane at the highest energies, and to discover around one hundred new supernova remnants and many hundreds of pulsar wind nebulae, according to current population estimates. The ability of the observatory to resolve such a large number of Galactic sources is one of the challenges to be faced. In this paper, we will present the first simulated scan of the Galactic plane with a realistic observation strategy, with particular attention to the potential source confusion. We will also present prospects for morphological studies of extended sources, such as the young SNR RX J1713.7-39.

35th International Cosmic Ray Conference ICRC2017

10-20 July, 2017

Bexco, Busan, Korea

\footnotetext{
*Speaker.

${ }^{\dagger}$ Max-Planck Institut ur Kernphysik, Heidelberg (Germany)

$\ddagger$ University of Delaware, US
} 
The Cherenkov Telescope Array (CTA) is a next-generation imaging atmospheric Cherenkov telescope (IACT) array that has been designed to detect photons from $20 \mathrm{GeV}$ up to $300 \mathrm{TeV}$. Currently under construction, CTA consists of two distinct arrays which will provide complete coverage of the entire sky. CTA-North will be located on the Canary island of La Palma, while CTASouth will be at the Paranal site in Chile. CTA will consist of multiple telescopes of three different sizes, each of which is optimized for a specific energy range. The largest telescopes (large-sized telescopes, LSTs), with a reflector surface of $23 \mathrm{~m}$ diameter, will provide an energy threshold of few tens of $\mathrm{GeV}$, whereas the smallest (4 m diameter small-sized telescopes, SST) guarantee the coverage of the highest energies up to hundreds of TeV. Medium-sized telescopes ( $12 \mathrm{~m}$ diameter, MST) provide unprecedented sensitivity in the central energy range. The performance of the two arrays is described in detail in $[1,2]$.

\section{The CTA Galactic Plane Survey}

One of the most important legacies of CTA will undoubtedly be its Galactic plane survey (GPS). This aims to provide the first complete very-high-energy (VHE) scan of the entire Galactic plane with a sensitivity better than $4 \mathrm{mCrab}$ for unresolved sources. The GPS will provide a complete census of the VHE gamma-ray sources in our Galaxy, as well as a precise characterization of the diffuse emission in the $0.1-100 \mathrm{TeV}$ energy range from the entire Galactic plane. Given the significant improvement in the performance of CTA with respect to current IACTs, this dataset will likely also reveal many new and unexpected phenomena, such as new classes of gamma-ray emitters and new types of transient and variable source behaviour.

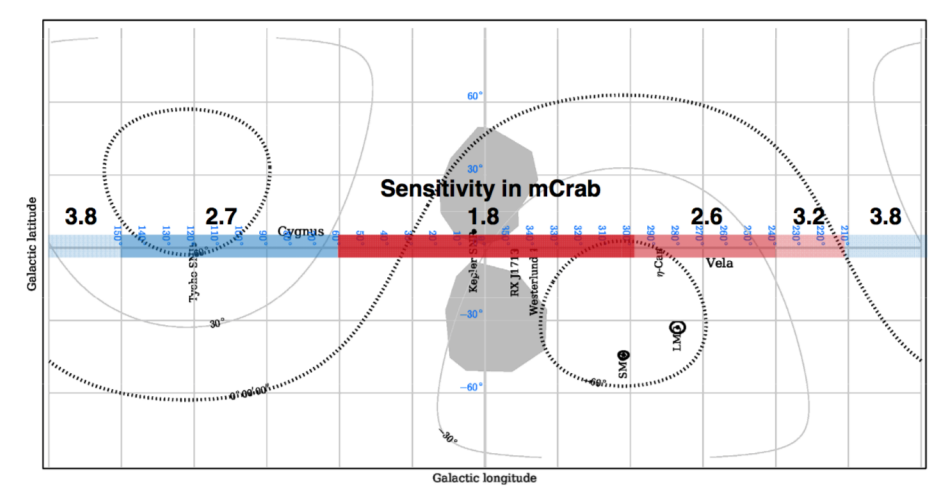

Figure 1: Point-source sensitivity in mCrab foreseen for the 10-year GPS program along the Galactic plane. Some known VHE objects are labelled for reference. Figure taken from [3].

The survey will be carried out using both the northern and the southern arrays, and will provide complete coverage of the Galactic plane. The exposure time will be non-uniform across the plane, and will be determined by the scientific goals to be addressed in different regions. The inner Galactic region $\left(60^{\circ}<l<60^{\circ}\right)$, for instance, will be allocated significantly more observation time than other regions, allowing to reach a sensitivity as low as $1.8 \mathrm{mCrab}$. GPS is divided into an earlyphase (1-2 years) and a long term-program (3-10 years) with a total of 1020 and $600 \mathrm{hr}$ requested 
for CTA-South and CTA-North, respectively. A double-row pointing strategy will be used, with a nominal separation distance of $3^{\circ}$ and the observational pattern will be set to optimize sensitivity to periodic signals on different time scales. Figure 1 shows the different point-like sensitivities which will be reached by the full 10-year program in the various regions of the sky.

The current generation of ground-based instruments has revealed that more than half of the detected TeV sources cluster along the Galactic plane ( $90 \%$ of the Galactic sources lie at $|\mathrm{b}|<2^{\circ}$ ). Pulsar wind nebulae are the most numerous class, followed by supernova remnants [4]. However, half of the Galactic plane sources remain unidentified for three main reasons: 1) they have multiple associations at lower energies which cannot be disentangled, 2) they consist of several still unresolved sources, 3) they are completely "dark", with no counterpart at any other wavelength. Counting both firm identifications and candidate associations, we find that $60 \%$ of the Galactic plane sources are PWNe-like' ${ }^{1}$, and 20-25\% are SNR-like.

Through the $\log N-\log S$ distribution of the VHE known sources, taken from the open-source gamma-cat catalogue [5], we can estimate the number of VHE sources expected to be detected by CTA. Figure 2 illustrates the obtained $\log N-\log S$, i.e. the cumulative source count as a function of the integral flux between 1 and $10 \mathrm{TeV}$. This predicts less than 100 SNRs and $400 \mathrm{PWNe}$. These numbers provide only an estimate of the number of sources expected to be detected by CTA for two main reasons: 1) the models consider point-like sensitivities whereas the sensitivity worsens for extended sources (as shown in [2]) 2) they are based on an extrapolation of the number of sources detected by the current generation of IACTs.

Figure 3 shows a realistic simulated image of the GPS obtained from observations with the southern array. This simulation includes all of the known VHE sources together with models for both the PWN and SNR populations, and for the diffuse emission. In order to preserve the total number of PWNe and SNRs, in agreement with the estimations derived above through the $\log N$ $\log S$ distribution, for each known source we removed from the corresponding model one source with a comparable integral flux. The SNR model, taken from [6], accounts only for young SNR - no interaction with the interstellar medium is foreseen at this stage. The PWN model was built with a phenomenological approach, similar to that described in [7]. In particular, we considered the radial distribution of the surface density of pulsars in the Galaxy taken from [8]. The intrinsic size was randomly generated by using a Gaussian probability density function, truncated at 0 . The differential energy spectra were assumed to be log-parabola functions, with the index and curvature parameters were randomly generated by using Gaussian probability density functions derived from the corresponding distributions of the known PWNe [9, 10], whereas the luminosity is estimated from a power-law density function with a slope of about one. We also coupled one-third of the PWN with the SNR to create a sample of composite SNRs with a variable ratio between the radius of the two components.

The Galactic interstellar medium diffuse model is based on predictions from codes that solve the cosmic ray (CR) transport equations and calculate the related multiwavelength emission. It includes emission from interstellar gas, produced by hadronic interactions of cosmic ray nuclei and by electron/positron Bremsstrahlung. It also accounts for the inverse-Compton (IC) radiation produced by the interactions of cosmic ray electrons and positrons with low-energy photons. We use predictions

\footnotetext{
${ }^{1}$ In this definition PWNe include composite SNRs
} 


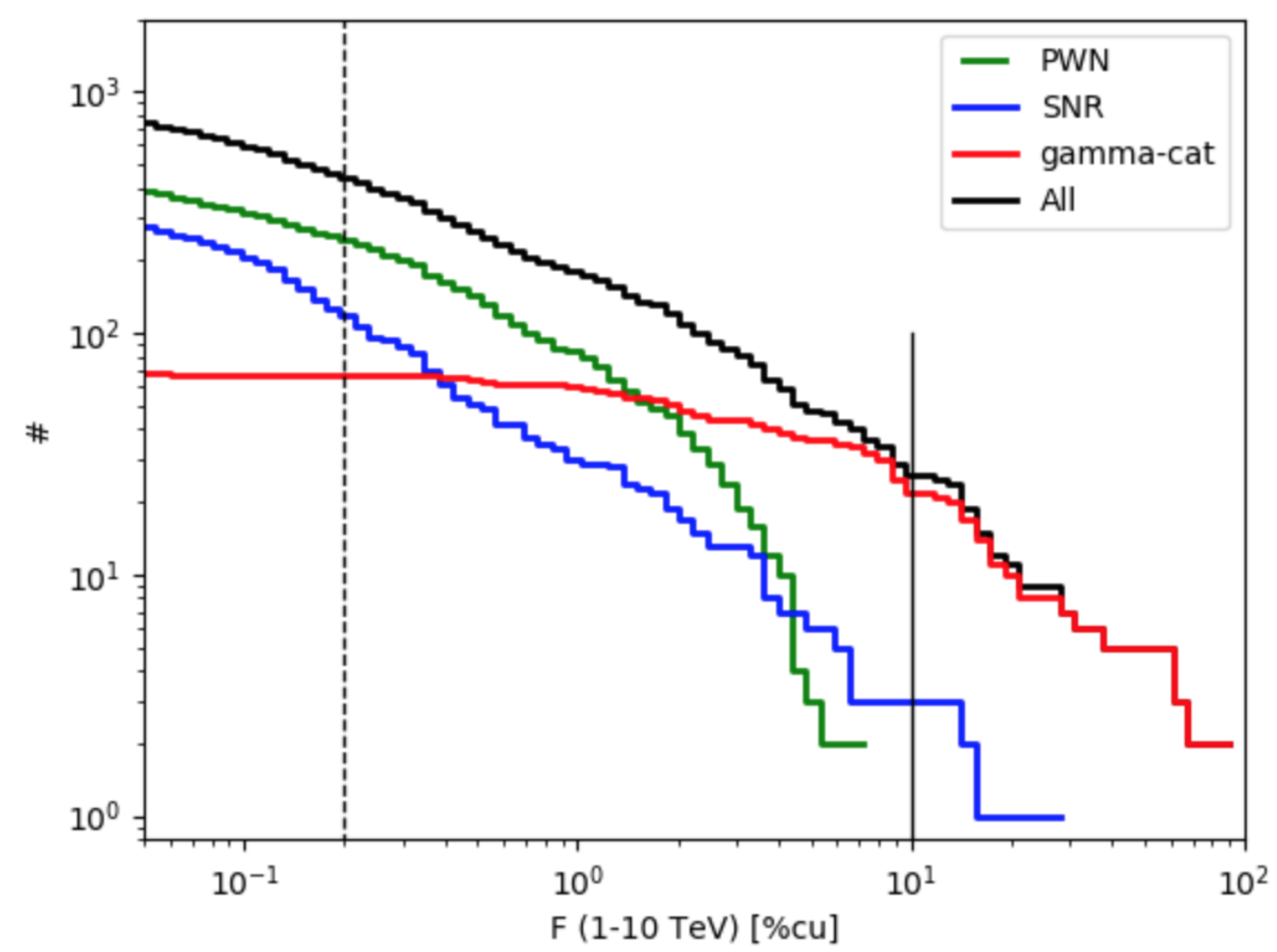

Figure 2: $\log N-\log S$ distribution. The red and black lines shows the known VHE sources taken from [5] and the VHE sources expected to be detected by CTA. Green and blue show the PWN and SNR populations, respectively, with the number of already known sources subtracted. The black vertical line indicates the completeness of the H.E.S.S. GPS at $10 \%$ of the Crab Nebula flux, whereas the dashed line shows the CTA expected completeness, at $0.2 \%$ of the Crab Nebula flux.

from Dragon [11], that assume position-dependent diffusion and convection properties to reproduce the intensity and spectral hardening of interstellar gamma-ray emission seen by Fermi-LAT toward the inner Galaxy. The resolution of the gas maps has been enhanced with respect to those used internally in Dragon by using the dust maps from Planck and IRAS, which have an angular resolution of $0.03^{\circ}$. The IC component was obtained using predictions from Picard, that assume a model with 4 spiral arms for the CR source distribution [12]. Figure 4 shows the count map of the obtained diffuse model.

This simulation of the GPS shows that source confusion is the most limiting factor in the detection of sources, especially in the innermost regions of the Galaxy where the source density can approach 3-4 sources per square degree. Preliminary studies, not yet considering the diffuse emission, have set lower limits to the estimated confusion from $13-24 \%$ at $100 \mathrm{GeV}$ to $9-18 \%$ at $1 \mathrm{TeV}$ for the region $|l|<30^{\circ}[3]$. 

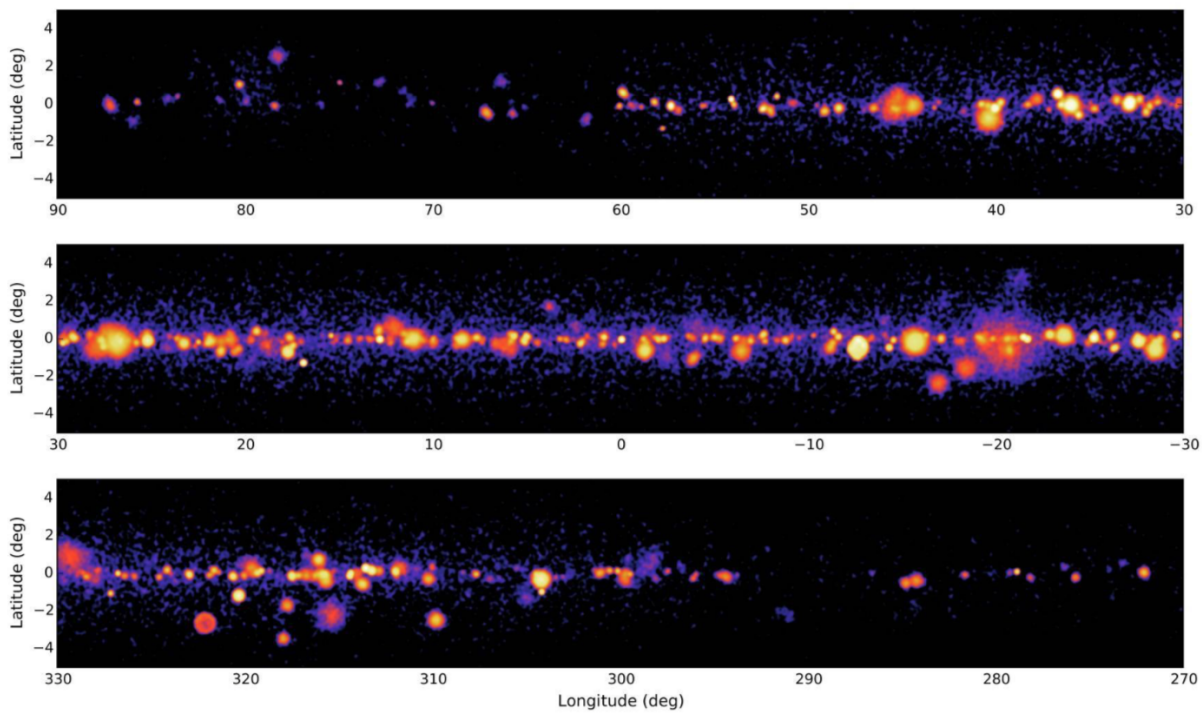

Figure 3: Simulated image of the Galactic plane survey carried out from the southern array within the 10-year program. These maps have not been produced by using the skymodel described in this proceeding.

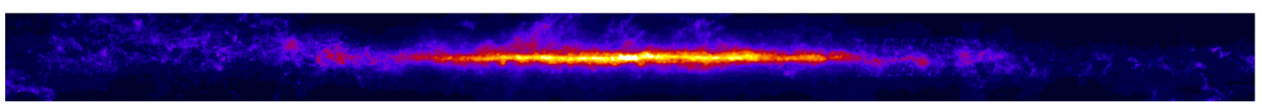

Figure 4: The Galactic interstellar medium diffuse model including both emission from the interstellar gas and the inverse Compton component.

\section{Morphological Capabilities}

The majority of the known VHE gamma-ray sources in the Galaxy are spatially extended, with no clear sub-structures and a typical angular size of $0.1-0.2^{\circ}$ [4]. This may be explained, in part, by observational biases, since the existing IACT arrays have relatively limited fields of view (up to $5^{\circ}$ ). The new results from HAWC, taking advantage of the larger field of view of this air shower array, show that almost half of the HAWC-detected Galactic sources have an angular size larger than $0.7^{\circ}$ [13]. CTA, with an angular resolution as low as $0.05^{\circ}$ at $1 \mathrm{TeV}$ [2] and a maximum FoV of $10^{\circ}\left(8^{\circ}\right)$ in the southern (northern) array is a perfect instrument to perform morphological studies of extended sources and to resolve structures down to arcminute scales. We show the capabilities of CTA for morphological studies by looking at the specific case of the bright young SNR, RX J1713.7-3946 [14]; one of the key targets for solving the problem of the CR origin. We created a template for our simulations by combining a leptonic component traced by the XMM Xray image, and a hadronic component based on $\mathrm{CO}$ and $\mathrm{HI}$ observations [15]. Figure 5 compares the latest results obtained by H.E.S.S. with those expected for $50 \mathrm{hr}$ of observations with CTA, in the two extreme cases of leptonic and hadronic dominant gamma-ray emission. As quantitatively proven in [15], CTA will be able to distinguish between the two scenarios. 

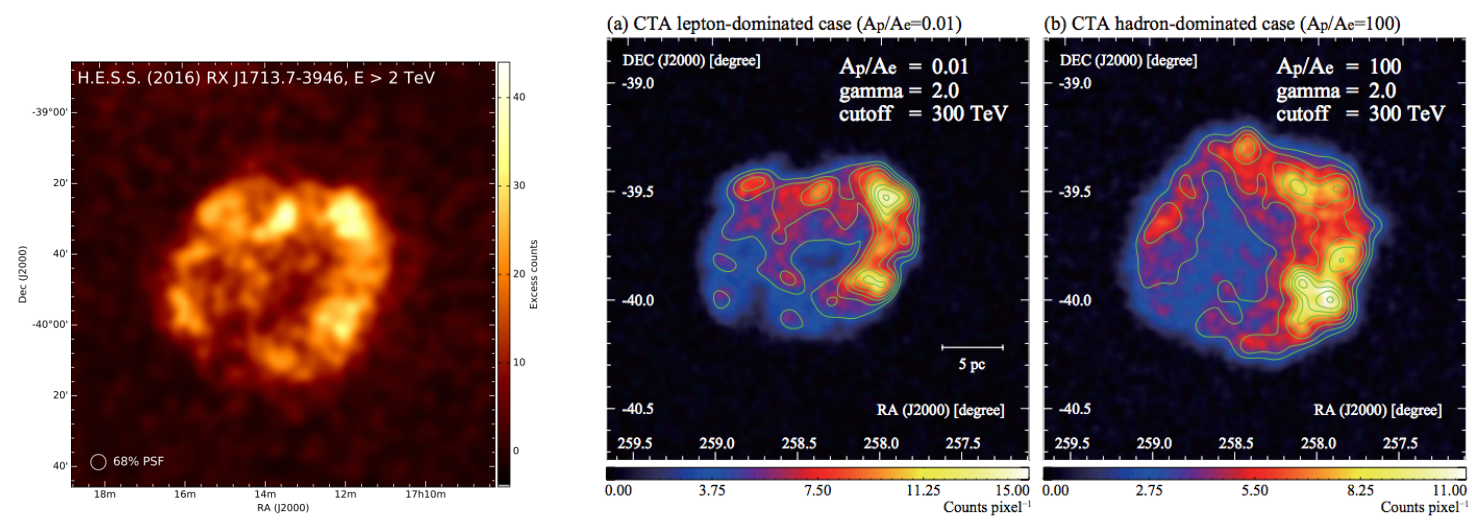

Figure 5: Image of RX J1713.7-3946. On the right H.E.S.S. result above $2 \mathrm{TeV}$ taken from [14]; On the left expected CTA results for the leptonic and hadronic dominated gamma-ray emission [15].

\section{Potential for Spectral Studies}

CTA's broadband coverage, together with its excellent energy resolution $(0.04 \%$ at $10 \mathrm{TeV}$ [1]), will allow to detect previously unmeasurable spectral features in Galactic sources. These could include extra spectral components, such in the case of RXJ1713.7-3946. In the leptonicdominant scenario of the emission from this young SNR, CTA may detect a flatter hadronic component emerging at very high energies, above $50 \mathrm{TeV}$ (see Figure 6), if the parent CR population is accelerated to more than $100 \mathrm{TeV}$. According to [15], such a detection, at $3 \sigma$ level, would require $50 \mathrm{hr}$ of observation by the southern CTA array. Another example is given by the gamma-ray spectra of molecular clouds illuminated by CRs from a nearby accelerator. In this case, a characteristic V-shape may be imprinted on the spectral energy distribution, due to the superposition of the gamma-ray emission from background CRs with that from the nearby source [16, 17].

\section{Conclusions}

The CTA Galactic plane survey will lead to a significant improvement in the understanding of our Galaxy at $\mathrm{TeV}$ energies, and will allow to perform spectral and morphological studies with unprecedented precision. CTA can resolve spatial sub-structures on arcminute scales, as well as reconstruct previously unmeasurable spectral features. The combination of these new capabilities will be particularly powerful, in that it provides the opportunity for spatially resolved spectroscopy in the VHE gamma-ray regime. This is a new window in $\mathrm{TeV}$ astronomy, which has previously required observation exposures of typically hundreds of hours. In addition to superbly detailed studies of specific, relatively bright objects, the GPS will provide a new catalogue of VHE sources which will increase the source count by a factor 3-9, and will very likely reveal new classes of Galactic gamma-ray emitters.

\section{Acknowledgments}

The CTA Consortium gratefully acknowledges financial support from the following agencies 

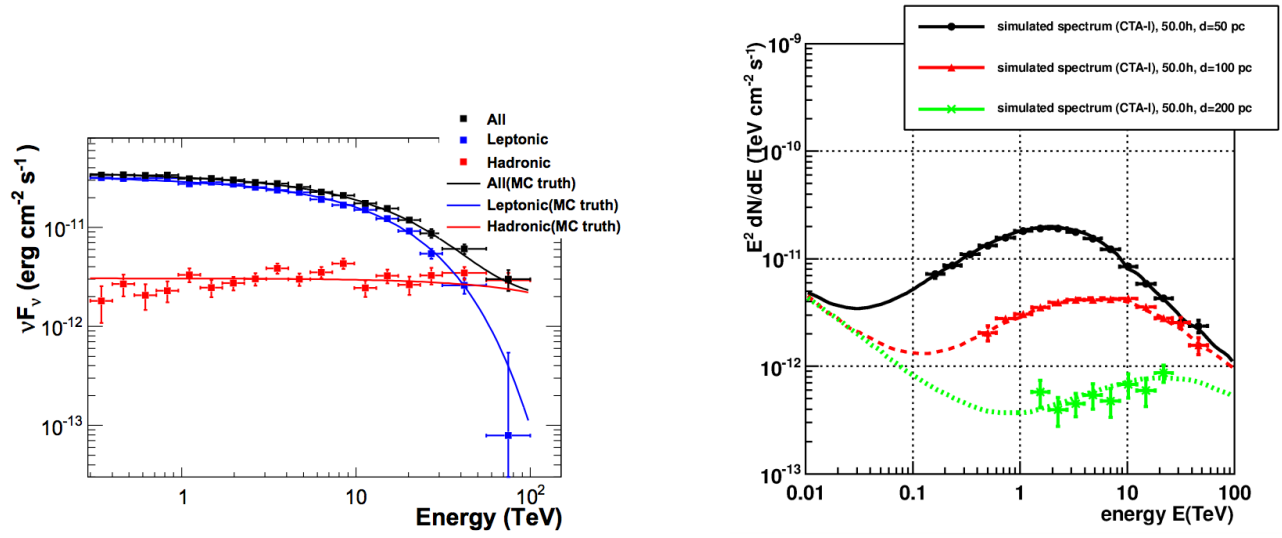

Figure 6: On the left: Spectral energy distribution of RX J1713.7-39. Blue and red squares are the spectral points for the leptonic and hadronic templates obtained for $50 \mathrm{hr}$ of observation. Black points are the total flux, the sum of the two components with error bars obtained by summing the two errors in quadrature. No systematic uncertainties are taken into account. Taken from [15]. On the right: Simulated spectral energy distribution of a molecular cloud illuminated by a nearby SNR obtained with $50 \mathrm{hr}$ of observations. Different colours refer to different distances between the cloud and the SNR. The molecular cloud is assumed to have a $10^{5} \mathrm{M}_{\odot}$ and lie at $1 \mathrm{kpc}$, whereas the SNR is $2000 \mathrm{yr}$ old. Taken from [17].

and organizations listed here: $h \mathrm{ttp}: / /$ www.cta-observatory.org/consortium_acknowledgments

\section{References}

[1] Bernlohr, K. et al. Astropart. Physics 43, 171 (2013)

[2] Gernot, M., Arrabito, L., Bernlhor, K., et al. These proceedings.

[3] The CTA Consortium, Science with the Cherenkov Telescope Array, to be submitted, July 2017.

[4] Donath, A. for the H.E.S.S. collaboration in Gamma 2016, AIP Conference Proceedings (2016)

[5] https://gamma-cat.readthedocs.io/

[6] Cristofari, P., Gabici, S., et al. These proceedings.

[7] Renaud, M. for the CTA consortium in Memorie della Societa Astronomica Italiana, v.82 (2011)

[8] Yusifov, I. \& Kucuk, I., A\&A 422 (2004)

[9] Kargaltsev, O., Rangelov, B., \& Pavlov, G. The Universe Evolution. Astrophysical and Nuclear Aspects", 2013, Nova Science Publishers

[10] M. Mayer PhD Thesis http://www-library.desy.de/cgi-bin/showprep.pl?desy-thesis-13-033

[11] Gaggero, D., Urbano, A., Valli, M., \& Ullio, P. Phys.R. D 91 (2015)

[12] Werner, M., Kissmann, A., Strong, A.W., Reimer, O., accepted in Astrop. Phys.

[13] HAWC collaboration, submitted to The Astrophys. Journal

[14] H.E.S.S. collaboration, accepted in A\&A arXiv:1609.08671

[15] CTA consortium, accepted in ApJ. arXiv:1704.04136 
[16] Gabici, S., Aharonian, F., \& Casanova, S. MNRAS 396, 1629 (2009)

[17] Acero, F. et al. Astropart. Physics 42, 276 (2013)

[18] Kelner, S., Aharonian, F. \& Bugayov, V. Phys.Rev D 74, 3 (2006)

[19] Romoli, C., Taylor, A., \& Aharonian, F. Astropart. Physics 88, 38 (2017) 